\title{
La législation européenne contre les discriminations et le devoir de diligence des EMN
}

\section{Silvia Borelli}

\section{(2) OpenEdition}

1 Journals

Édition électronique

URL : https://journals.openedition.org/rdctss/1990

DOI : 10.4000/rdctss. 1990

ISSN : 2262-9815

Éditeur

Centre de droit comparé du travail et de la sécurité sociale

\section{Édition imprimée}

Date de publication : 1 avril 2018

Pagination : 58-73

ISSN : 2117-4350

\section{Référence électronique}

Silvia Borelli, « La législation européenne contre les discriminations et le devoir de diligence des

EMN », Revue de droit comparé du travail et de la sécurité sociale [En ligne], 1 | 2018, mis en ligne le 01 novembre 2021, consulté le 12 novembre 2021. URL : http://journals.openedition.org/rdctss/1990 ; DOI : https://doi.org/10.4000/rdctss. 1990

\section{(c) (†) $९$}

Revue de droit comparé du travail et de la sécurité sociale est mise à disposition selon les termes de la Licence Creative Commons Attribution - Pas d'Utilisation Commerciale - Pas de Modification 4.0 International. 


\title{
LA LÉGISLATION EUROPÉENNE CONTRE LES DISCRIMINATIONS ET LE DEVOIR DE DILIGENCE DES EMN*
}

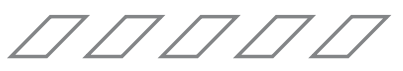

\section{RÉSUMÉ}

Les principes de non-discrimination sont des règles contraignantes qui interdisent de traiter un individu ou un groupe caractérisé par un ou plusieurs facteurs énumérés par la loi anti-discrimination d'une manière qui produit ou peut produire un désavantage. La discrimination est normalement imputée à la personne qui exerce le pouvoir qui a produit ou peut produire une discrimination. Cependant, une personne peut également être responsable de discriminations lorsqu'elle a toléré illégalement un tel acte. C'est le cas lorsqu'une personne a le devoir d'empêcher les discriminations de se produire. Les sources de l'obligation de prévenir les discriminations peuvent être inscrites dans le devoir de diligence d'une entreprise vis-à-vis d'une autre. A partir de là, cet article discute de la possibilité de poursuivre une société européenne (c'est-à-dire une société ayant son siège ou un établissement en Europe) pour des actes de discrimination commis par ses filiales, ses fournisseurs ou ses sous-traitants sur le territoire de l'UE ou hors de celui-ci. En analysant les différentes sources nationales, européennes et internationales, l'auteur montre comment l'interconnexion entre la législation anti-discrimination de I'UE et le devoir de diligence peut être utile pour faire respecter un devoir de diligence juridiquement non contraignant et pour garantir aux victimes des recours efficaces.

\section{MOTS CLÉS : Législation anti-discrimination, devoir de diligence, responsabilité.}

\begin{abstract}
The principles of non-discrimination are binding rules that forbid treating an individual or a group characterised by one or more factors listed by antidiscrimination law in a way that produces or can produce a disadvantage. Discrimination is normally ascribed to the person who has exerted the power that has produced or can produce the discriminatory effect. However, a person can be liable for discrimination as well when she/he has illegally tolerated an act of discrimination. This is the case when a person has a duty to prevent discrimination to happen. The sources of the duty to prevent discrimination to happen can be enshrined in the duty of care of one company over a different one. Based on this conceptual framework, the paper discusses the possibility to sue a European company (i.e. a company that has its headquarters or a unit in Europe) for acts of discrimination caused by its subsidiaries, suppliers or subcontractors inside or outside the EU territory. Distinguishing among national, European and international sources, the author demonstrates how the interconnection between EU Antidiscrimination Law and the duty of care can be helpful to enforce a non-legally binding duty of care and to guarantee to victims effective remedies.
\end{abstract}

\section{KEYWORDS : Antidiscrimination Law, Duty of Care, Liability.}

* Une première version de cet article a été présentée lors de la Conférence du Réseau de recherche en droit du travail (LLRN), à Toronto, du 25 au 27 juin 2017. L'auteur remercie Isabelle Daugareilh et les autres participants pour leurs commentaires. 
es principes de non-discrimination sont à l'origine de règles contraignantes qui interdisent de traiter un individu ou un groupe caractérisé par un ou plusieurs facteurs énumérés par la législation anti-discrimination de telle sorte que cela produit ou peut produire un désavantage. Lorsqu'il examine un cas présumé de discrimination, le tribunal doit vérifier si une disposition, un critère ou une pratique a produit ou peut produire une inégalité de traitement, si l'inégalité de traitement est objectivement justifiée par un but légitime et si les moyens d'atteindre ce but sont appropriés et nécessaires (dans le cas d'une discrimination directe, le juge devra simplement vérifier si l'une des exceptions prévues par la législation anti-discrimination s'applique). On peut considérer une disposition, un critère ou une pratique comme une forme d'exercice du pouvoir, qu'il s'agisse d'un pouvoir privé ou public, d'un pouvoir juridique ou factuel. En conséquence, à travers les principes de non-discrimination, le juge peut examiner toute forme d'exercice du pouvoir par rapport aux effets qu'il peut produire ou qu'il a produits' ${ }^{1}$.

Les directives anti-discrimination de I'UE n'indiquent pas qui devrait être tenu pour responsable de la discrimination : celui qui exerce un pouvoir dans le champ d'application des directives est tenu de respecter l'interdiction de discrimination qui y est établie. En conséquence, l'auteur de l'acte de discrimination à lıégard dıun salarié ou dıun groupe de salariés n'est pas nécessairement l'employeur ${ }^{2}$. La responsabilité de la discrimination est normalement attribuée à la personne qui a exercé le pouvoir qui a produit ou peut produire l'effet discriminatoire. Cependant, selon la législation anti-discrimination de l'UE, la personne (physique ou morale) qui a agi de manière discriminatoire n'est pas considérée comme la seule responsable. Tout d'abord, l'effet discriminatoire peut être le résultat d'une pluralité de décisions. La législation anti-discrimination de l'UE prévoit, par exemple, le cas où une instruction est donnée de discriminer ${ }^{3}$.

La CJUE n'a pas encore précisé si une société mère peut être conjointement et solidairement responsable avec l'une de ses filiales pour la violation de la législation anti-discrimination de I'UE. En revanche, la CJUE a déclaré qu'une société mère est conjointement et solidairement responsable avec les autres personnes morales constituant une unité économique unique des infractions au droit de la concurrence. "Même si la société mère ne participe pas directement à linfraction, elle exerce, dans une telle hypothèse, une influence déterminante sur les filiales qui ont participé à celle-ci ${ }^{4}$ »

1 M. Barbera, Discriminazioni ed eguaglianza nel rapporto di lavoro, Giuffré, Milan, 1991, 51.

2 CJEU, 9 octobre 2001, C-379/99, Menauer, paragraphes 29 et 30.

3 Article 2, paragraphe 4, directive 2000/43 / CE, article 2, paragraphe 4, directive 2000/78 / CE, article 2, paragraphe 2 de la directive 2006/54 / CE

4 CJUE, 10 septembre 2009, C-97/08, Akzo Nobel NV, paragraphe 77. 
Il convient de souligner qu'une personne (morale ou physique) peut être considérée comme l'auteur d'une discrimination, non seulement dans les cas où elle a exercé activement son pouvoir, mais même dans les cas où elle a toléré illégalement un acte de discrimination. Une omission peut donc constituer un acte de discrimination lorsque la personne responsable a le devoir de l'empêcher. Par exemple, une agence de travail temporaire peut être considérée comme responsable d'une discrimination à l'encontre des travailleurs intérimaires pour des pratiques d'une entreprise utilisatrice car, en tant qu'employeur de travailleurs intérimaires, elle a le devoir « d'assurer la sécurité et la santé des travailleurs pour tous les aspects liés au travail. ${ }^{5}$ »

Sur la base de ce cadre conceptuel ${ }^{6}$, cet article s'intéresse à la question de savoir si et quand une société européenne (c'est-à-dire une société qui a son siège ou un établissement en Europe) peut être poursuivie pour des actes de discrimination causés par ses filiales, fournisseurs ou sous-traitants sur le territoire de I'UE ou en dehors de celui-ci. A cette fin, nous examinerons d'abord le contenu des obligations de l'entreprise visant à prévenir les violations des droits de l'homme par ses filiales, fournisseurs ou sous-traitants, établis au niveau international, européen et national (I). Ensuite, nous montrerons comment I'interconnexion entre ces devoirs et la législation anti-discrimination de l'UE peut être utile pour faire respecter des devoirs de vigilance non juridiquement contraignants et pour garantir aux victimes de discrimination des recours efficaces (II).

\section{I - LE DEVOIR DES EMN DE RESPECTER LES DROITS DE L'HOMME ET DE FAIRE PREUVE DE DILIGENCE RAISONNABLE EN DROIT INTERNATIONAL ET EUROPÉEN}

\section{A - LE DROIT INTERNATIONAL}

Selon les "Principes directeurs relatifs aux entreprises et aux droits de l'homme: mise en œuvre du cadre de référence "protéger, respecter et réparer » des Nations Unies ", les entreprises devraient éviter "d'avoir des incidences négatives sur les droits de l'homme ou d'y contribuer par leurs propres activités $»^{7}$ et devraient prévenir ou atténuer «les incidences négatives sur les droits de l'homme qui sont directement liées

5 Article 5, paragraphe 1 de la directive 89/391 / CEE.

6 Voir S. Borelli, "Principles of Non-Discrimination" in E. Ales, F. Basenghi, W. Bromwich e I. Senatori, Giappichelli (dir.) Complex Economic Organisations, Employment Relations and Transformation of the Enterprise in the Global Economy, Giappichelli, Torino, 2015, p. 3.

7 Ce devoir inclut également les activités de l'entreprise dans la chaîne d'approvisionnement (Principes directeurs de l'OCDE à l'intention des entreprises multinationales, Commentaire sur les principes généraux, paragraphe 17, dorénavant Principes directeurs de I'OCDE). 
à leurs activités, produits ou services par leurs relations commerciales, même si elles n'ont pas contribué à ces incidences " (principe $\left.n^{\circ} 13\right)^{8}$. Le terme "relations commerciales» inclut les relations «avec des partenaires commerciaux, des entités appartenant à la chaîne d'approvisionnement ou toute autre entité, publique ou non, directement liée à ses activités, ses produits ou ses services ${ }^{9} "$.

Des obligations similaires ont été introduites lors de la révision des Principes directeurs de l'OCDE en 2011 qui a étendu ces obligations à toutes les questions couvertes par les Principes directeurs ${ }^{10}$. Les entreprises devraient également poursuivre un processus de diligence raisonnable en matière de droits de l'homme, "identifier leurs incidences sur les droits de l'homme, prévenir ces incidences et en atténuer les effets, et rendre compte de la manière dont elles y remédient ${ }^{11} »$. Le processus de diligence raisonnable « devrait viser les incidences négatives sur les droits de l'homme que l'entreprise peut avoir ou auxquelles elle peut contribuer par le biais de ses propres activités, ou qui peuvent découler directement de ses activités, produits ou services par ses relations commerciales ${ }^{12}$ ».

Comme précisé dans les Principes des Nations Unies, les mesures à prendre varient en fonction de la marge de manœuvre de l'entreprise ${ }^{13}$. On considère que cette marge de manœuvre ou influence existe "lorsque l'entreprise a la capacité d'apporter des changements aux pratiques illicites d'une entité qui commet un abus ${ }^{14}$ ». Si une entreprise identifie un risque de contribuer à la réalisation d'une incidence négative, " elle doit prendre les mesures nécessaires pour empêcher ou faire cesser sa contribution et user

8 Les principes directeurs de I'ONU s'appliquent à tous les Etas et à toutes les entreprises, transnationales et autres, indépendamment de leur taille, localisation, propriété et structure. Parmi les droits de l'homme que les entreprises commerciales doivent respecter, figurent les droits garantis par les huit conventions fondamentales de I'OIT énoncées dans la Déclaration de I'OIT relative aux principes et droits fondamentaux au travail (principe $n^{\circ} 12$ ). La Convention $n^{\circ} 111$ concernant la discrimination en matière d'emploi et de travail et la Convention sur l'égalité de rémunération ( $n^{\circ} 100$ ) figurent parmi les huit conventions fondamentales de I'OIT.

9 Principes directeurs de l'OCDE, Commentaires sur les principes généraux, paragraphe 14 et Commentaire sur les droits de l'homme, paragraphe 43 ; Principes directeurs relatifs aux entreprises et aux droits de l'homme : mise en œuvre du cadre de référence «Protéger, respecter et réparer » des Nations Unies », Commentaire sur le principe $n^{\circ} 13$.

10 II. Principes généraux, paragraphes 11 et 12, et IV. Droits de l'homme, paragraphes 2 et 3.

11 Principes des Nations Unies, principe $n^{\circ} 15$, b), et Principes directeurs de I'OCDE, II. Principes généraux, paragraphe 10, qui étendent la diligence raisonnable à toutes les questions couvertes par les principes directeurs, et IV. Droits de l'homme, par. 5.

12 Principes des Nations Unies, principe $n^{\circ} .17$.

13 Principes des Nations Unies, principe $n^{\circ} 19$.

14 Principes des Nations Unies, Commentaire sur le principe $n^{\circ} 19$, et Principes directeurs de l'OCDE, Commentaires sur les principes généraux, paragraphe 19 et Commentaire sur les droits de I'homme, paragraphe 42. 
de son influence pour atténuer les incidences restantes dans la mesure du possible $\|^{15}$. II faut souligner qu'une entreprise peut contribuer aux incidences négatives pour les droits de l'homme causées par une autre partie lorsqu'elle bénéficie « de l'atteinte commise par ladite partie ${ }^{16}$.

Lorsqu'une entreprise n'a pas contribué à une incidence négative mais que celleci est directement liée à ses opérations, produits ou services réalisés par une relation commerciale, elle doit utiliser "son influence pour intervenir auprès de l'entité responsable de l'incidence négative afin de prévenir ou atténuer cette incidence ${ }^{17}$.

Des obligations similaires de respect des droits de l'homme et de diligence raisonnable ont été introduites lors de la révision en 2017 de la Déclaration de principes tripartite de I'OIT sur les entreprises multinationales et la politique sociale qui les renvoie aux EMN ${ }^{18}$. Le terme EMN est utilisé dans la Déclaration de l'OIT pour désigner " diverses entités (société mère, entité locale ou les deux, ou encore l'ensemble du groupe), entre lesquelles se répartissent les responsabilités, dans l'idée qu'elles coopéreront et s'entraideront, le cas échéant, pour être mieux à même d'observer les principes énoncés " dans la Déclaration de l'OIT (objet et champ d'application, paragraphe 6). La Déclaration de I'OIT stipule également que «le degré d'autonomie de chaque entité par rapport aux autres au sein des entreprises multinationales est très variable d'une entreprise à l'autre, selon la nature des liens qui unissent ces entités et leur domaine d'activité et compte tenu de la grande diversité qui caractérise les entreprises concernées du point de vue des formes de propriété, de la taille, de la nature des activités et du lieu d'implantation " (objet et champ d'application, paragraphe 6$)^{19}$. Par conséquent, lorsqu'une filiale commet une violation des droits de I'homme et n'a pas (ou très peu) d'autonomie, cette violation doit être attribuée à la société mère. En outre, tous les devoirs régis par la Déclaration de l'OIT (y compris l'égalité des chances et de traitement) sont attribués aux EMN. Cela signifie que la société mère est obligée conjointement avec les filiales de respecter ces devoirs et qu'elle est conjointement responsable de toute violation, sauf lorsque la filiale a agi de manière autonome.

15 Principes des Nations Unies, Commentaire sur le principe n. 19, et Principes directeurs de I'OCDE, Commentaire sur les principes généraux, paragraphe 19 et Commentaire sur les droits de I'homme, paragraphe 42. "Contribuer à » une incidence négative doit être interprété comme une contribution substantielle, c'est-à-dire une activité qui provoque, facilite ou incite une autre entité à provoquer une incidence négative » (Commentaire sur les principes généraux, paragraphe 14).

16 Principes des Nations Unies, Commentaire sur le principe $n^{\circ}$ 17. M.-C. Caillet, «Le devoir de vigilance et les relations d'affaires : vers une responsabilité collective? », I. Daugareilh, La responsabilité sociale de l'entreprise, vecteur d'un droit de la mondialisation?, Bruylant, 2017, p. 515.

17 Principes directeurs de I'OCDE, Commentaire sur les principes généraux, paragraphe 20, et Principes des Nations Unies, Commentaire sur le principe $n^{\circ}$ 19. "Les réponses appropriées peuvent prendre plusieurs formes : poursuite de la relation avec le fournisseur pendant toute la durée des efforts d'atténuation des risques; suspension temporaire de la relation avec poursuite des efforts d'atténuation des risques; ou, en dernier recours, rupture de la relation avec le fournisseur, soit après que les efforts d'atténuation des risques ont échoué, soit parce que l'entreprise estime qu'aucune atténuation n'est possible, soit à cause de la gravité de l'incidence négative » (Principes directeurs de l'OCDE, Commentaire sur les principes généraux, paragraphe 22).

18 Politique générale, paragraphe 10, c) et d).

19 Sur l'influence et le contrôle que les entreprises dirigeantes exercent sur les opérations de leurs fournisseurs et sous-traitants, voir OIT, Le travail décent dans les chaînes d'approvisionnement mondiales, Rapport IV, Conférence internationale du Travail, 105ème session, 2016. 
Selon les Principes directeurs de l'OCDE à l'intention des entreprises multinationales, pour ce qui est des entreprises multinationales, « il s'agit généralement d'entreprises ou d'autres entités établies dans plusieurs pays et liées de telle façon qu'elles peuvent coordonner leurs activités de diverses manières. Une ou plusieurs de ces entités peuvent être en mesure d'exercer une grande influence sur les activités des autres; leur degré d'autonomie au sein de l'entreprise peut être très variable d'une multinationale à l'autre ${ }^{20}$ ». Les Principes directeurs de l'OCDE s'adressent à toutes les entités de la multinationale qui sont censées coopérer et se prêter mutuellement concours pour faciliter le respect des lignes directrices, " en fonction de la répartition effective des responsabilités entre elles ${ }^{21}$ ". Les Principes directeurs de l'OCDE exigent également des sociétés mères qu'elles assurent «le pilotage stratégique de l'entreprise, et en contrôlent efficacement la direction ${ }^{22}$ ». Les systèmes de conformité et de contrôle devraient s'étendre autant que possible aux filiales. Enfin, «la mission de surveillance des pratiques de gouvernance qui incombe au conseil implique également de contrôler en permanence les structures internes d'un groupe afin de s'assurer que les responsabilités y sont clairement définies partout ${ }^{23}$ ».

Un processus de diligence raisonnable (c'est à dire " une démarche d'identification, visant à éviter ou atténuer les impacts négatifs sociaux, environnementaux et économiques réels et potentiels, qui résultent des décisions et activités d'une organisation " dans l'ensemble de la sphère d'influence de l'entreprise) est également indiqué dans la liste des enjeux concernant l'amélioration de la responsabilité sociétale par l'ISO $26000^{24}$. L'ISO 26000 fait référence à la sphère d'influence de l'entreprise, c'est-à-dire la portée «des relations politiques, contractuelles, économiques ou autre à travers lesquelles une organisation a la capacité d'influer sur les décisions ou les activités de personnes ou d'autres organisations $»^{25}$.

Toutes les sources examinées ici sont des documents juridiquement non contraignants, c'est-à-dire que leur respect par les entreprises est facultatif. En effet, il n'existe actuellement aucun instrument international juridiquement contraignant pour réglementer les activités des sociétés transnationales et autres entreprises ${ }^{26}$.

20 I. Concepts et principes, paragraphe 4.

21 I. Concepts et principes, paragraphe 4.

22 Commentaire sur les principes généraux, paragraphe 8.

23 Commentaire sur les principes généraux, paragraphe 9.

24 Article 7.3.1. Voir I. Daugareilh, L'ISO à l'assaut du social : risques et limites d'un exercice de normalisation sociale, I. Daugareilh (dir.), Responsabilité sociale de l'entreprise transnationale et globalisation de l'économie, Bruylant, Bruxelles, 2010, p. 563.

25 ISO 26000: 2010, Article 2.19.

26 En 2014, le Conseil des droits de l'homme des Nations Unies a créé un groupe de travail intergouvernemental pour un traité contraignant sur les entreprises transnationales et autres entreprises en matière de droits de l'homme. De nombreux Etats membres européens (dont I'Autriche, la France, l'Allemagne et l'Italie) et les Etats-Unis ont voté contre cette décision. En 2016, le Parlement européen a invité les Etats membres à soutenir l'initiative des Nations unies (résolution du 25.10.2016 sur la responsabilité des entreprises pour les violations graves des droits de l'homme dans les pays tiers). Voir J. Martens et K. Seitz, The Struggle for a UN Treaty Towards global regulation on human rights and business, Global Policy Forum and Rosa Stiftung-New York Office, Foundation, 2016, B. Faracik, Implementation of the UN Guiding Principles on Business and Human Rights, Etude pour la sous-commission des droits de l'homme du Parlement européen, Bruxelles, 2017, p. 18. 


\section{B - LES DEVOIRS DE DILIGENCE À L'ÉCHELLE EUROPÉENNE}

Au niveau européen, la directive 2014/95/UE a introduit pour les « grandes entreprises qui sont des entités d'intérêt public " de plus de 500 salariés et pour les " entités d'intérêt public qui sont les entreprises mères d'un grand groupe " de plus de 500 salariés, l'obligation de publier une déclaration non financière sur le développement, la performance, la position et l'impact de l'activité de l'entreprise du groupe sur les questions " environnementales, sociales, de personnel, de respect des droits de l'homme et de lutte contre la corruption ${ }^{27}$ ». La Déclaration comprend à la fois " une description des politiques appliquées par le groupe en ce qui concerne ces questions, y compris pour les procédures de diligence raisonnable mises en œuvre " et " les principaux risques liés à ces questions en rapport avec les activités du groupe, y compris, lorsque cela s'avère pertinent et proportionné, les relations d'affaires, les produits ou les services du groupe, qui sont susceptibles d'entraîner des incidences négatives dans ces domaines, et la manière dont le groupe gère ces risques ${ }^{28}$ ". Le processus de diligence raisonnable devrait identifier, prévenir et atténuer les incidences négatives existantes et potentielles qui " peuvent découler des propres activités de l'entreprise ou peuvent être liés à ses activités et, lorsque cela s'avère pertinent et proportionné, à ses produits, à ses services et à ses relations d'affaires, y compris ses chaînes d'approvisionnement et de sous-traitance $~_{29}$. En fournissant cette information, l'entreprise peut s'appuyer sur des cadres internationaux tels que les Principes des Nations Unies, les Principes directeurs de I'OCDE, la Déclaration de I'OIT ou la norme ISO 26000 (Considérant $\left.n^{\circ} 9\right)^{30}$.

Un système de diligence raisonnable est également exigé par le règlement de l'Union européenne sur le bois ${ }^{31}$. Dans ce contexte, la diligence raisonnable exige qu'un opérateur entreprenne un exercice de gestion des risques de manière à minimiser le risque de mise sur le marché de I'UE d'un bois récolté illégalement ou de produits dérivés contenant du bois récolté illégalement.

Un règlement européen sur les minerais provenant de zones de conflitvisant à introduire des obligations de diligence raisonnable pour les entreprises de l'UE afin d'atténuer le risque de violations des droits de l'homme tout au long de la chaîne d'approvisionnement a été adoptée en $2017^{32}$. Les importateurs de minerais et de métaux de l'UE doivent respecter le devoir de diligence à l'égard de la chaîne d'approvisionnement conformément aux normes énoncées dans le Guide de l'OCDE sur le devoir de diligence pour des chaînes

27 Art. 19a et 29a, paragraphe 1 de la directive 2013/34/UE.

28 Art. 19a et 29a, paragraphe 1 Directive 2013/34 / UE. «En ce qui concerne les questions sociales et de personnel, les informations fournies dans la Déclaration peuvent porter sur les mesures prises pour garantir lıégalité hommes-femmes, la mise en œuvre des conventions fondamentales de l, Organisation internationale du travail (OIT), les conditions de travail, le dialogue social, le respect du droit des travailleurs à être informés et consultés, le respect des droits syndicaux, la santé et la sécurité sur le lieu de travail, le dialogue avec les communautés locales et/ou les mesures prises en vue de garantir la protection et le développement de ces communautés " (considérant $n^{\circ} 7$, Directive 2014/95/UE).

29 Considérant $n^{\circ} 8$.

30 Communication de la Commission, Lignes directrices sur l'information non financière (méthodologie pour la communication d'informations non financières), 2017/C 215/01, par. 4.2.

31 Règlement (UE) n 995/2010.

32 Règlement 2017/821. 
d'approvisionnement responsables en minerais provenant de zones de conflit ou à haut risque (article 4). Les mesures de gestion des risques à adopter pour prévenir ou atténuer les incidences négatives dépendent de la capacité de l'entreprise «à exercer une influence et, au besoin, des pressions sur les fournisseurs les plus à même de prévenir ou d'atténuer efficacement les risques mis en lumière $»^{33}$.

\section{II - LE DEVOIR DE DILIGENCE À L'ÉCHELLE NATIONALE}

Au niveau national, il faut d'abord citer la récente loi française sur le devoir de vigilance des sociétés mères et des entreprises donneuses d'ordre (Loi n 2017-399) ${ }^{34}$. Cette loi oblige toute entreprise établie en France qui emploie au moins 5000 salariés au siège social et dans ses filiales directes et indirectes, et toute entreprise qui emploi au moins 10000 salariés au siège social et dans ses filiales directes et indirectes, dont le siège social est situé sur le territoire français ou à l'étranger, à établir et à mettre en œuvre de manière effective un plan de vigilance ${ }^{35}$. Ce plan doit inclure des mesures appropriées pour identifier les risques et pour prévenir les atteintes graves aux droits de l'homme et aux libertés fondamentales, à la santé et la sécurité des personnes et l'environnement, résultant des activités de l'entreprise et de celles des sociétés qu'elle contrôle directement ou indirectement, ainsi que des activités des sous-traitants et des fournisseurs avec lesquels elle entretient une relation commerciale établie ${ }^{36}$. En cas de violation de devoirs de diligence, la société est tenue pour responsable et doit réparer le préjudice que l'exécution de ces obligations aurait permis d'éviter ${ }^{37}$.

33 Art. 5, paragraphe 1, alinéa b) (ii).

34 Sur le droit français, voir N. Cuzacq, Le devoir de vigilance des sociétés mères et des entreprises donneuses d'ordre, Dalloz, Paris, 2015 ; R.C. Drouin, "Le développement du contentieux à l'encontre des entreprises transnationales : quel rôle pour le devoir de vigilance ? ", Droit Social, 2016 , p. 246 ; O. Favereau, « Le devoir de vigilance dans les groupes et réseaux de sociétés. Sur la proposition de loi adoptée par l'Assemblée nationale le 30 mars 2015 ", Revue Droit du Travail, 2015, p. 446-450; C. Hannoun, "Pour un dispositif de vigilance mesuré et efficace», Revue Droit Travail, 2014, pp. 441-444; C. Hannoun, Proposition pour un devoir de vigilance des sociétés mères, in Mélanges offerts à M. Germain, Dalloz, Paris, 2014, p. 342 ; A. Lyon-Caen et T. Sachs, "La responsabilité des entreprises multinationales. Un processus de constitutionnalisation en action ", in J.P. Robé, A. Lyon-Caen and S. Vernac (dir.), Multinationals and the constitutionalization of the world power system, Routledge, New York, 2016, p. 204 ; M. Moreau, Le rôle des juges face à l'exigence d'une réglementation sociale transnationale, Droit social, n. ${ }^{\circ} 3$, p. 2016 ; S. Schiller, "Encourager plutôt que contraindre », Revue Droit du Travail,2014, pp. 444-446; Sachs T., "La loi sur le devoir de vigilance des sociétés mères et sociétés donneuses d'ordre : les ingrédients d'une corégulation », Revue Droit Travail, 2017, p. 380, et les essais publiés dans Droit social, n 10/2017.

35 Art. L. 225-102-4 du Code du commerce.

36 Pour les détails sur le contenu du plan, voir l'art. L. 225-102-4 al. 4 du Code du commerce français.

37 Art. 225-102-5 CP. Le Conseil constitutionnel français a déclaré non conformes à la Constitution la sanction civile de 10 à 30 millions d'euros pour manquement à l'obligation de diligence (décision n. 2017-750). 
Un processus de diligence raisonnable est également prévu par la loi sur l'esclavage moderne au Royaume-Uni (UK Modern Slavery Act) de 201538. Cette loi oblige toute organisation qui exploite une entreprise (ou une partie d'une entreprise) au RoyaumeUni à élaborer une déclaration sur l'esclavage et la traite des êtres humains (c'est-à-dire une déclaration sur les mesures prises par l'organisation pour s'assurer qu'il n'y a aucun esclavage et aucune traite des êtres humains dans ses chaînes d'approvisionnement), qui peut inclure ses processus de diligence raisonnable concernant l'esclavage et la traite des êtres humains dans ses activités et ses chaînes d'approvisionnement ${ }^{39}$. La jurisprudence anglaise établit également que la société mère peut, dans certaines circonstances, avoir un devoir de diligence envers les salariés d'une filiale ${ }^{40}$. L'obligation de diligence de la société mère repose sur trois conditions (le test Caparo sur la négligence $)^{41}$ :

1) le dommage est prévisible,

2) il existe suffisamment de proximité entre les parties,

3) l'existence d'un tel devoir de diligence est équitable, juste et raisonnable.

Si les trois conditions sont remplies, la société mère est déclarée " directement " responsable. Dans le cas Chandler v Cape plc [2012] EWCA Civ 525, la Cour d'Appel a jugé que la société mère était responsable de l'inexécution de son devoir de diligence à l'égard des salariés d'une filiale souffrant de maladies professionnelles liées à l'exposition à l'amiante parce qu'elle savait ou aurait dû se rendre compte que les conditions de travail dans la filiale étaient dangereuses et qu'elle aurait dû prévoir le risque de dommages (prévisibilité). En outre, la société mère exerçait un contrôle suffisant sur la filiale, puisqu'elle avait pris des mesures de santé et de sécurité applicables à tous les salariés du groupe, y compris les salariés de ses filiales (proximité entre le demandeur et la société). Enfin, le fait que l'exposition à l'amiante soit reconnue comme un risque majeur pour la santé constituait un motif juste et raisonnable pour imposer l'obligation de diligence ${ }^{42}$.

Enfin, il convient de mentionner la Résolution concernant le travail décent dans les chaînes d'approvisionnement mondiales, adoptée par la Conférence générale de l'OIT à sa 105e session (2016). Dans ce document, il est recommandé aux Etats de « stimuler la transparence et encourager les entreprises, et le cas échéant de les contraindre, par divers moyens à faire rapport sur la diligence raisonnable dans leurs chaînes d'approvisionnement

38 Voir D. Blackburn, A. Sanders, R. Subasinghe, Supply chains liability: UK report, 2015.

39 Modern Slavery Act 2015, partie 6, art. 54, par. 5.

40 R. Meeran, "Access to Remedy: the United Kingdom Experience of MNC Tort Litigation for Human Rights Violations", in S Deva et D Bilchitz (dir), Human Rights Obligations of Businesses: Beyond the Corporate Responsibility to Respect?, CUP, 2013; A Sanger, "Crossing the corporate veil: the duty of care owed by a parent company to the employees of its subsidiary" CLJ, 2012, n71, p.478; Bright C., "Le devoir de la société mère dans la jurisprudence anglaise", Droit Social, 2017, p. 828.

41 Caparo Industries Plc v Dickman [1990] 2 AC 605 (HL).

42 Selon L.J. Arden, les circonstances dans lesquelles la loi peut imposer à une société mère la responsabilité de la santé et de la sécurité des salariés de sa filiale « comprennent une situation où, comme en l'espèce, (1) les activités de la société mère et de la filiale sont le même (2) la société mère a, ou devrait avoir, des connaissances supérieures sur certains aspects pertinents de la santé et de la sécurité dans l'industrie en question, (3) le système de travail de la filiale est dangereux et la société mère le savait, ou aurait dû savoir, et (4) la société mère savait ou aurait dû prévoir que la filiale ou ses salariés compteraient sur l'utilisation de cette connaissance supérieure pour la protection des salariés » (Chandlerv Cape plc [2012] EWCA Civ 525, [80]). 
et à faire connaître la façon dont elles gèrent les incidences de leur activité sur les droits de I'homme ${ }^{43}$ ». De même, les Principes directeurs de l'OCDE engagent les pays adhérant à la Déclaration de l'OCDE sur l'investissement international et les entreprises multinationales à promouvoir l'application des Principes directeurs par toutes les entreprises ayant leur siège dans leur pays, où qu'elles exercent leur activité, ainsi que par les entreprises opérant sur leur territoire. Comme indiqué dans le Rapport du Haut-Commissaire des Nations Unies aux droits de l'homme Améliorer la responsabilisation des entreprises et l'accès à des voies de recours pour les victimes de violations des droits de l'homme, "Garantir la responsabilisation juridique des entreprises et l'accès à des voies de recours utiles pour les personnes victimes de ces violations est une composante fondamentale de l'obligation des États de protéger contre les atteintes aux droits de l'homme commises par des entreprises » (paragraphe 1).

\section{III - LE DEVOIR DE DILIGENCE DANS LES ACCORDS-CADRES INTERNATIONAUX}

Une obligation de diligence tout au long de la chaîne d'approvisionnement peut également être réglementée par des accords-cadres globaux négociés entre les syndicats et les entreprises multinationales. Actuellement, ni le droit international ni le droit européen ne réglementent ces accords de sorte qu'ils ne sont généralement pas considérés comme juridiquement contraignants.

L'Accord Danone/UITA sur l'emploi durable et l'accès aux droits signé le 15 mars 2016, par exemple, oblige Danone à encourager l'application de l'accord chez les fournisseurs du groupe et dans les activités auxquelles Danone participe, selon le devoir de vigilance tel que régulé par les Principes directeurs de I'OCDE ${ }^{44}$. L'accord garantit également un salaire égal pour un travail égal et des conditions de travail non discriminatoires pour les travailleurs directement employés par Danone, les travailleurs intérimaires et les travailleurs employés par un prestataire de services.

Cependant, la majorité des accords ne font pas référence à une obligation de diligence raisonnable, mais introduisent simplement différents types de devoirs pour la société mère afin d'encourager le respect des droits des travailleurs par d'autres sociétés impliquées dans ses activités. L'accord-cadre mondial entre H\&M, Industrial Global Union et Industrifacket Metall sur le respect et la mise en œuvre des normes internationales du travail chez les fournisseurs de $\mathrm{H}$ \& M signé le 9 septembre 2015 impose par exemple à H \& M « d'utiliser toute son influence potentielle pour s'assurer que ses fournisseurs directs et leurs soustraitants produisant des marchandises ou des produits vendus dans tous les magasins de détail du groupe H \& M respectent les droits humains et syndicaux sur le lieu de travail ».

L'accord européen 2012 du Groupe GDF Suez sur l'égalité professionnelle entre les femmes et les hommes engage l'entreprise à s'assurer que ses fournisseurs et sous-traitants respectent les principes d'égalité entre les femmes et les hommes (art. 13). De même,

43 Par.16 (f) et (i). Voir également la Recommandation du Comité des Ministres du Conseil de l'Europe sur les droits de l'homme et les entreprises, adoptée le 2 mars 2016, paragraphe 20.

44 Sur l'Accord Danone/UITA, voir M. Frapard, " La protection des droits sociaux fondamentaux des travailleurs dans les entreprises transnationales », Revue de Droit du Travail, 2017, p. 49. 
l'accord-cadre international entre ThyssenKrupp AG, le comité de groupe IG Metall et IndustriALL Global Union signé en 2015 oblige l'entreprise à encourager ses fournisseurs à prendre en compte les principes fondamentaux et les normes fondamentales du travail de l'OIT (art. 10) dans leur politique d'entreprise. Cependant, les deux accords ne précisent pas quelles mesures doivent être adoptées pour remplir ces devoirs.

Enfin, nous devrions citer les codes de conduite et les initiatives de conformité engagées volontairement par les sociétés mères pour surveiller le respect des droits de l'homme. Nonobstant les limites des initiatives volontaires pour identifier et prévenir efficacement les risques ${ }^{45}$, ces mécanismes volontaires impliquent parfois un devoir de vigilance sur la chaîne d'approvisionnement ou bien font référence au respect des Principes directeurs des Nations Unies, des Principes directeurs de l'OCDE ou de la Déclaration de I'OIT.

\section{IV - LE DEVOIR DE DILIGENCE ET LA RESPONSABILITÉ EN MATIÈRE DE DISCRIMINATION}

Après avoir brièvement cité les différentes sources du devoir de l'entreprise de prévenir les violations des droits de l'homme par ses filiales, fournisseurs ou sous-traitants, nous devons vérifier si et quand une entreprise européenne peut être tenue pour responsable d'une discrimination dans sa chaîne d'approvisionnement. Les prémisses de notre analyse sont :

1. le fait qu'une entreprise de la chaîne d'approvisionnement ait eu une pratique discriminatoire contre ses travailleurs pour un motif interdit par la législation antidiscrimination de l'UE (préjudice),

2. le fait que la société mère ait enfreint son devoir de diligence dans la chaîne d'approvisionnement (manquement au devoir),

3. le fait que le devoir de diligence concerne le respect du principe de nondiscrimination violé par l'entreprise dans la chaîne d'approvisionnement,

4. le fait qu'ayant respecté l'obligation de diligence, l'entreprise mère aurait pu empêcher ou atténuer la discrimination dans la chaîne d'approvisionnement (lien de causalité entre le dommage et le manquement au devoir).

Bien sûr, dans une affaire en instance de jugement, tous ces postulats doivent être prouvés et ce n'est pas souvent une tâche facile. Afin de faire respecter le devoir de diligence, de nombreux auteurs ont réclamé des mesures pour inverser le fardeau de la preuve, c'est-à-dire exiger des entreprises mères qu'elles démontrent qu'elles ont pris toutes les mesures raisonnables pour éviter les dommages ou qu'elles n'avaient aucun

45 Voir Rapport mondial de Human Rights Watch, Without Rules : A Failed Approach to Corporate Accountability, 2013 ; AFL-CIO, Responsibility Outsourced: Social Audits, Workplace Certification and Twenty Years of Failure to Protect Worker Rights, 2013. 
contrôle sur les activités qui ont causé le préjudice ${ }^{46}$. En outre, il a été suggéré d'introduire une obligation de divulgation qui permettrait aux plaignants de demander au tribunal d'ordonner à une société défenderesse de divulguer tous les détails concernant le contrôle exercé sur ses filiales et sous-traitants, son implication générale dans la gestion de ses filiales et sous-traitants, son contrôle et son implication dans le cas spécifique lié à la réclamation, dans la mesure où cette information est pertinente pour apprécier le devoir de diligence de la société-défenderesse ${ }^{47}$.

Comme précisé ci-dessus, il n'existe pas encore un général devoir de diligence juridiquement contraignant au niveau international ou européen. En effet, chaque règlement peut lier différentes entreprises (portée subjective), peut fournir une définition différente de la relation commerciale (portée objective), peut être appliqué à différents droits de l'homme, peut être juridiquement contraignant ou non. II existe cependant un élément commun entre ces devoirs : les mesures que la société mère doit adopter pour prévenir ou réduire le risque de violation des droits de l'homme dépendent du degré d'influence qu'elles ont sur leur chaîne d'approvisionnement.

Le lien entre la législation anti-discrimination de l'UE et le devoir de vigilance peut permettre d'attribuer la responsabilité de la discrimination à l'entreprise dominante : comme nous l'avons déjà souligné, une entreprise est responsable d'une discrimination qu'elle a le devoir de prévenir. D'un autre côté, ce lien résout le problème de l'absence de force contraignante du devoir de diligence : la violation du devoir de diligence est une prémisse de la violation de la loi anti-discrimination de l'UE. Par conséquent, les dispositions non contraignantes sont utilisées comme base pour "la reconnaissance d'obligations découlant de règles de droit dur, contribuant de ce fait à la construction d'un système de régulation mixte $»^{48}$.

46 J. J. Álvarez Rubio, K. Yiannibas (dir.), Human Rights in Business. Removal of barriers to access to justice in the European union, Routledge, 2017, IV; Improving access to remedy in the area of business and human rights at the EU level, Avis de l'Agence des droits fondamentaux de I'Union européenne, 2016, p. 7 et 34; S. Cossare et M.L. Guislain, « Le devoir de vigilance pour les entreprises multinationales, un impératif juridique pour une économie durable », RLDA, 2015, p. 75. En interprétant la loi française sur le devoir de diligence, C. Hannoun (« Le devoir de vigilance des sociétés mères et entreprises donneuses d'ordre après la loi du 27 mars 2017 », Droit Social, 2017, p. 816) a suggéré d'appliquer aux sociétés mères la jurisprudence sur la "présomption d'identification du risque en cas d'alerte " : si la société mère est alertée sur certains risques, elle a le devoir d'enquêter et de prendre des mesures appropriées.

47 C. van Dam, F. Gregor, Corporate responsibility to respect human rights. Vis-à-vis legal duty of care, dans Human Rights in Business. Removal of barriers to access to justice in the European Union. Executive summary, 2017, p. 22.

48 R.C. Drouin, «Le développement du contentieux à l'encontre des entreprises transnationales : quel rôle pour le devoir de vigilance? », Droit Social, 2016, p. 255. Voir aussi K. Martin-Chenut, « Devoir de vigilance : inter-normativités et durcissement de la RSE», Droit Social, 2017, p. 802. 
Tenir compte de l'obligation de diligence raisonnable (même si elle n'est pas juridiquement contraignante pour les entreprises transnationales) dans l'évaluation de la responsabilité en matière de discrimination contribuerait à faire respecter les droits de l'homme par les entreprises, comme l'exigent les Principes des Nations Unies ${ }^{49}$, les Principes directeurs de l'OCDE ${ }^{50}$ et la Déclaration de l'OIT ${ }^{51}$.

Lorsque l'obligation de diligence est issue d'un accord-cadre mondial ou d'un code de conduite, les tribunaux devraient tenir compte du fait que l'entreprise dominante a volontairement signé cet accord ou adopté le code, en s'engageant dans un processus de diligence raisonnable. Par conséquent, la violation du devoir de diligence par cette entreprise (même s'il n'est pas contraignant) devrait être prise en compte et permettre de lui imputer la responsabilité de la discrimination dans sa chaîne d'approvisionnement.

Lorsqu'il existe un devoir de diligence juridiquement contraignant, l'avantage de lier la législation anti-discrimination de I'UE au devoir de diligence réside dans la possibilité dıutiliser les mesures d'exécution réglementées par le premier (par exemple, le renversement de la charge de la preuve, l'action collective, les procédures d'urgence, les sanctions), pour prouver et sanctionner les discriminations survenues dans la chaîne d'approvisionnement.

Si la discrimination a été causée par une filiale, un fournisseur ou un sous-traitant établi dans un Etat différent de celui où la société mère est établie, il est nécessaire de déterminer le tribunal compétent et la loi applicable. Les litiges concernant les Etats membres de I'UE sont gouvernés par le règlement Bruxelles I bis et le règlement Rome II. Le premier prescrit que « les personnes domiciliées sur le territoire d'un État membre sont attraites, quelle que soit leur nationalité, devant les juridictions de cet État membre $\aleph^{52}$. Autrement, la société défenderesse peut être poursuivie « devant la juridiction du lieu où le fait dommageable

49 Les Principes des Nations Unies obligent les Etats à «énoncer clairement qu'ils attendent de toutes les entreprises domiciliées sur leur territoire et/ou sous leur juridiction qu'elles respectent les droits de l'homme dans toutes leurs activités » (principe $\mathrm{n}^{\circ} 2$, voir également Recommandation du Comité des Ministres du L'Europe sur les droits de l'homme et les entreprises, adoptée le 2 mars 2016, par. 13).

50 Les Principes directeurs de l'OCDE exigent que les gouvernements encouragent les entreprises opérant sur leur territoire à respecter les règles établies ici, quel que soit le lieu où elles opèrent (Principes directeurs de l'OCDE, I. Concepts et principes, par. 3).

51 La Déclaration de l'OIT prescrit aux gouvernements de veiller à ce que, lorsque des violations des droits de l'homme se produisent sur leur territoire et/ou dans leur juridiction, tout travailleur ou groupe de travailleurs concerné ait accès à un recours effectif (par. 64). Les juridictions nationales, faisant partie de l'organisation de l'Etat, remplissent les obligations de l'Etat mentionné.

52 Art. 4, par. 1 du règlement $n^{\circ}$ 1215/2012. Aux fins du règlement $n^{\circ}$ 1215/2012, «les sociétés et les personnes morales sont domiciliées là où est situé : a) leur siège statutaire, (b) leur administration centrale; ou (c) leur principal établissement » (Article 63, paragraphe 1). Voir O. De Schutter O., "La responsabilité des Etats dans le contrôle des sociétés transnationales : vers une convention internationale sur la lutte contre les atteintes aux droits de l'homme commises par les sociétés transnationales ", in I. Daugareilh I. (dir.), Responsabilité sociale de l'entreprise transnationale et globalisation de l'économie, Bruylant 2010, p. 707. 
s'est produit ou risque de se produire ${ }^{53}$. Par conséquent, la société mère peut être poursuivie devant le tribunal de l'Etat membre où elle est établie ou devant le tribunal de l'Etat membre où la discrimination s'est produite. Devant le tribunal où est poursuivie la société mère, il est également possible de poursuivre la filiale, le fournisseur ou le soustraitant établi dans un autre Etat membre ${ }^{54}$.

Le règlement Rome II affirme que « la loi applicable à une obligation non contractuelle résultant d'un fait dommageable est celle du pays où le dommage survient ${ }^{5}$ ". Par conséquent, il convient d'appliquer la loi du pays où la discrimination a eu lieu. Toutefois, l'article 16 prévoit que le tribunal compétent applique les « dispositions de la loi du for qui régissent impérativement la situation, quelle que soit la loi applicable à l'obligation non contractuelle ». La législation nationale mettant en œuvre la législation anti-discrimination de I'UE peut figurer parmi les dispositions impératives dérogatoires de la loi du for, ainsi que la législation nationale sur le devoir de diligence ${ }^{56}$. En effet, l'article 16 fait référence à des dispositions d'importance fondamentale telles que celles adoptées pour mettre en $œ u v r e$ les normes fondamentales du travail de I'OIT, les droits et principes fondamentaux de l'UE et les dispositions dont l'objectif a été internationalement reconnu ${ }^{57}$.

53 Art. $7, n^{\circ} 2$ du règlement $n^{\circ} 1215 / 2012$. Le lieu de l'évènement dommageable couvre à la fois le territoire du pays où l'évènement à l'origine du dommage est survenu et le territoire du pays où le résultat préjudiciable a eu lieu (CJUE, 30.11.1976, affaire 21/1976).

54 La révision du règlement Bruxelles I en 2012 n'a pas introduit le forum nécessitatis. Selon le Conseil de l'Europe, «les Etats devraient envisager d'autoriser leurs tribunaux internes à se déclarer compétents pour les requêtes civiles liées à des violations de droits de l'homme causées par cette entreprise en l'absence manifeste d'un autre forum garantissant un procès équitable (forum necessitatis) » (Conseil de l'Europe, Recommandation sur les droits de l'homme et les entreprises, 2.3.2016, paragraphe 36).Voir D. Augenstein, N. Jägers, Judicial remedies: the issue of jurisdiction, in Human Rights in Business. Removal of barriers to access to justice in the European Union. Executive summary, 2017, p. 13; Improving access to remedy in the area of business and human rights at the EU level, Avis de l'Agence des droits fondamentaux de I'Union européenne, 2016, page 8. Certains Etats membres maintiennent la compétence de nécessité (forum necessitatis) pour éviter les cas de déni de justice et garantir une protection judiciaire efficace telle que définie à l'article 6 de la Convention européenne des droits de l'homme (ECHR) (A. Pigrau Solé, M. Álvarez Torné, A. Cardesa-Salzmann, M. Font I Mas, D. Iglesias Márquez, J. Jaria I Manzano, Human rights in business. A practical handbook for civil society organisations and human rights defenders, 2016, p. 42 and p. 68 on). Sur le forum necessitatis voir l'affaire française Comilog: I. Bertrand, "Coemploi international : juridiction territorialement compétente », Revue de droit du travail, 2015, p. 203 ; E. Pataut, "Le contentieux collectif des travailleurs face à la mondialisation. Réflexions à partir de l'affaire Comilog », Droit Social, 2016, p. 554.

55 Article 4, paragraphe 1 du règlement $n^{\circ} 864 / 2007$.

56 Voir J. J. Álvarez Rubio et K. Yiannibas (eds.), Human Rights in Business. Removal of Barriers to Access to Justice in the European Union, Routledge, 2017, partie II ; O. De Schutter, "Le contrôle du respect des droits de l'homme par les sociétés transnationales : le rôle de l'État d'origine ", in H. Muir Watt, M.A. Moreau, P. Rodière, Justice et mondialisation en droit du travail. Du rôle du juge aux conflits alternatifs, Dalloz, Paris, 2010.

57 M. Saage-Maaß, Holding Companies Accountable. Lessons from transnational human rights litigation, 2014, p. 22 (https://www.brot-fuer-die welt.de/fileadmin/mediapool/2 Downloads/ Fachinformationen/Sonstiges/Brochure HoldingCompaniesAccountable Einzelseiten.pdf). 
Une autre possibilité est offerte par l'article 4, paragraphe 3, du règlement Rome II selon lequel, si le délit est manifestement plus étroitement lié à un pays autre que celui dans lequel le dommage est survenu, la loi de cet autre pays s'applique. «Un lien manifestement plus étroit avec un autre pays pourrait se fonder, notamment, sur une relation préexistante entre les parties » (art. 4, par. 3), comme c'est le cas entre la société mère et les filiales, fournisseurs ou sous-traitants ${ }^{58}$.

Les deux interprétations sont cohérentes avec l'obligation des Etats, en vertu des Principes directeurs des Nations Unies (principe $n^{\circ} 25$ ), d'envisager des moyens de réduire les obstacles juridiques, pratiques et autres qui pourraient conduire à un refus dıaccès à un recours juridique.

Par conséquent, lorsque la discrimination a eu lieu dans un Etat membre de I'UE, la société mère peut être poursuivie devant le tribunal de son lieu de domicile ${ }^{59}$. Ce tribunal doit appliquer la loi de l'Etat où le dommage est survenu ${ }^{60}$. La législation anti-discrimination étant harmonisée au niveau de l'UE, le tribunal compétent doit appliquer le droit national d'une manière compatible avec les critères mentionnés dans la première partie de cet article, c'est-à-dire doit déclarer la société mère responsable des discriminations qu'elle aurait pu empêcher en respectant son devoir de diligence.

Lorsque la société mère est établie dans un Etat membre de l'UE et que la discrimination s'est produite en dehors du territoire de l'UE, elle peut être poursuivie devant le tribunal de son lieu de domicile ${ }^{61}$ et cette juridiction devrait considérer que la loi anti-discrimination de I'UE fait partie des dispositions impératives de la lex fori. Dans ce cas également, une société mère établie dans un État membre de l,UE devrait être tenue responsable des discriminations survenues dans sa chaîne diapprovisionnement qu'elle aurait pu empêcher si elle avait respecté son devoir de diligence.

58 E. Pataut « Le devoir de vigilance. Aspects de droit international privé », Droit Social, 2017, p. 839.

59 Art. 4, par. 1 du règlement 1215/2012.

60 Art. 4, par. 1, règlement 864/2007.

61 Sur la possibilité des victimes de violations des droits de l'homme dans des pays tiers commis par des entreprises multinationales basées dans I'UE de les poursuivre devant les tribunaux, voir J. J. Álvarez Rubio et K. Yiannibas (dir.), Human Rights in Business. Removal of Barriers to Access to Justice in the European Union, Routledge, 2017, partie I. 


\section{Conclusion}

Les principes de non-discrimination de l'UE doivent être pris en compte parmi les techniques permettant de réguler l'exercice du pouvoir au-delà du contrat de travail et dans les organisations complexes : toute personne ayant causé ou toléré illégalement une discrimination en est responsable. Par conséquent, une entreprise peut être tenue pour responsable d'une discrimination causée par une autre entreprise dans sa chaîne d'approvisionnement, chaque fois que l'accomplissement du devoir de diligence aurait pu empêcher la discrimination de se produire.

L'établissement d'un lien entre la législation anti-discrimination de l'UE et le devoir de diligence permettrait de faire respecter un devoir de diligence non juridiquement contraignant : la violation du devoir de diligence devenant une partie du jugement antidiscrimination. En outre, les mesures d'exécution établies parla législation anti-discrimination de I'UE peuvent faciliter la collecte de preuves et la lutte contre les violations des droits de I'homme dans la chaîne d'approvisionnement. Par conséquent, l'interconnexion entre la soft law et la hard law renforce l'obligation, pour les multinationales, de respecter les droits de l'homme dans la chaîne globale d'approvisionnement. II en résulte une réaffectation des responsabilités au sein des organisations complexes : une entreprise mère est conjointement responsable de violations causées par ses filiales, fournisseurs ou soustraitants, lorsqu'elle a le pouvoir d'influencer les conduites risquées dans ses relations commerciales et qu'elle tire profit des activités de la chaîne d'approvisionnement.

\section{SILVIA BORELLI}

Professeur de droit du travail, Université de Ferrare (Italie)

Thèmes de recherche : Droit européen, droit antidiscriminatoire, droit du travail, organisations économiques complexes.

\section{Publications:}

S. Borelli, J.M. Serrano García, « El necesario reconocimiento de los derechos sindicales a los trabajadores de la economia digital $»$, Revista de Derecho Social, n 80/2018.

S. Borelli, "Tecniche di regolazione delle organizzazioni complesse e disciplina lavoristica", Lavoro e diritto, n 1/2014, pp. 23-35. 